\title{
Une paillardise rituelle
}

Les chants des captifs dioula

\section{Jean Derive}

\section{(2) OpenEdition}

\section{Journals}

Édition électronique

URL : https://journals.openedition.org/clo/645

DOI : $10.4000 /$ clo. 645

ISSN : 2266-1816

Éditeur

INALCO

Édition imprimée

Date de publication : 1 janvier 2009

Pagination : 259-286

ISBN : 978-2-85831-188-0

ISSN : 0396-891X

Référence électronique

Jean Derive, «Une paillardise rituelle », Cahiers de littérature orale [En ligne], 66 | 2009, mis en ligne le 25 février 2013, consulté le 01 juillet 2021. URL : http://journals.openedition.org/clo/645 ; DOI : https:// doi.org/10.4000/clo.645

Ce document a été généré automatiquement le 1 juillet 2021.

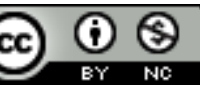

Cahiers de littérature orale est mis à disposition selon les termes de la Licence Creative Commons Attribution - Pas d'Utilisation Commerciale 4.0 International. 


\title{
Une paillardise rituelle
}

\author{
Les chants des captifs dioula
}

\author{
Jean Derive
}

\section{NOTE DE L'ÉDITEUR}

Article paru pour la première fois dans Cahiers de littérature orale, $\mathrm{n}^{\circ} 3,1977$, p. 105-133.

1 Les nomenclatures populaires des genres littéraires utilisent, pour les désigner, diverses sortes de procédés, qui impliquent différents points de vue dans la façon de les considérer. Le plus souvent, elles ont recours à plusieurs types de critères qui peuvent se combiner entre eux.

2 Tout d'abord des critères d'ordre textuel, susceptibles eux-mêmes d'être relatifs, soit à des propriétés formelles, soit à des propriétés de contenu.

3 Critères textuels formels: dans la plupart des cas, ces critères formels sont la base indispensable de la taxinomie et fonctionnent comme un trait de première position pour définir les genres. Précisons toutefois que le concept de «critères textuels » devra ici être entendu dans un sens très large, à partir du moment où l'on inclut la littérature orale. En effet, il peut alors aussi bien s'appliquer à des propriétés de l'énoncé (propriétés linguistiques) qu'à des propriétés de l'énonciation (propriétés paralinguistiques, telles qu'accompagnement musical et énonciation chantée, par opposition à énonciation parlée) ${ }^{1}$.

4 Critères textuels de contenu : ce second type de critères vient le plus souvent se combiner au premier, sous forme d'un déterminant qui donne une information supplémentaire sur une propriété du contenu: roman «d'aventures», roman "policier», conte « merveilleux», histoires «drôles», chansons "paillardes »... Bien entendu, l'état actuel des travaux sur le discours, et tout particulièrement sur les discours institutionnellement formalisés, a mis en évidence l'étroite corrélation qui existait entre forme et contenu, et notamment le fait que, dans bien des cas, le contenu déterminait une forme. 
5 Ainsi, il a pu être établi que le conte merveilleux, pour citer les célèbres travaux de Propp et de ses successeurs, correspondait à une forme bien particulière, à l'intérieur de la matrice générale « conte populaire ». On pourrait, dans le même esprit, esquisser un modèle structural du roman "policier » sous l'archétype formel «roman", ou de l'histoire " cochonne » sous celui de "l'histoire drôle ». Nous ne tombons donc pas dans l'illusion naïve qui consisterait à considérer, du point de vue d'une théorie du discours, forme et contenu comme deux propriétés juxtaposées de l'œuvre littéraire, parfaitement indépendantes l'une de l'autre. Nous constatons seulement ici que les taxinomies populaires, pour désigner les genres, combinent souvent dans leur terminologie une référence à la forme et une référence au contenu, comme si ces propriétés étaient simplement additives.

6 Toutefois, ce ne sont pas toujours des traits de contenu qui viennent s'ajouter aux traits formels pour définir les genres. Il arrive souvent qu'on ait recours à d'autres critères, notamment des critères circonstanciels, qui, eux, ne concernent par définition que le seul niveau de l'énonciation. Les circonstances auxquelles il est ainsi fait référence dans le nom du genre peuvent être des fêtes fixées par un calendrier rituel (chants ou contes de Noël), des événements plus aléatoires de la vie sociale, tels que certaines cérémonies (compliments ou chants de mariages, chants funèbres), manifestations (littérature de foire), activités (récits de chasse), ou tout simplement l'évocation d'un certain contexte socioculturel (théâtre de boulevard).

7 En général, à l'intérieur de la même culture, tous ces critères se chevauchent, ce qui montre que les points de vue varient pour désigner les genres littéraires. Un tel état de fait suggère que la rhétorique populaire a comme l'intuition que, selon les genres, les critères les plus pertinents pour les qualifier ne sont pas les mêmes.

8 Toutes ces généralités se vérifient pour la société des Dioula de Kong, fraction d'une ethnie africaine située au nord-est de la Côte d'Ivoire, dont nous allons parler plus particulièrement dans le cadre de cet article. Dans la taxinomie autochtone des genres de la littérature dioula, on trouve des termes qui font référence à des critères formels : un grand nombre de ces genres est désigné par le mot ḋ̀nkili qui signifie "chanson ", d'autres ont pris le nom de l'instrument qui accompagne le discours, comme le bàra, sorte de pot-pourri de contes, proverbes et chansons ; d'autres encore, comme le cúku cúku (chant guerrier) sont qualifiés par une sorte d'onomatopée reproduisant le rythme du tambour d'accompagnement.

9 À ces traits de première position peuvent s'associer, là aussi, des caractéristiques qui évoquent une propriété du contenu: ngálen kúma (contes étiologiques) signifient littéralement "parole d'autrefois », ce qui suppose une thématique liée aux origines; les kó kòro, récits historico légendaires, se traduisent par "affaire ancienne", expression qui évoque le caractère historique du discours; les lásiri dònkili (devises chantées) peuvent se décomposer mot à mot comme étant des " chants de généalogie ", ce qui renvoie encore à une thématique.

10 On trouve également toutes sortes de références circonstancielles dont nous avons parlé ; qu'il s'agisse :

- de fêtes à date fixe, comme les chants de masque (dò dònkili), qui portent chacun le nom spécifique du masque auquel ils sont attachés (sáfo dònkili, dòmuso dònkili...), et qui sont énoncés lorsque celui-ci sort, aux jours fixés par le calendrier rituel ;

- de cérémonies plus ou moins aléatoires : kónyon dònkili (chants de mariage) dénsagali dònkili (chants de baptême)...; 
- de références à des activités particulières, sèncke dònkili (chants agricoles) qui sont destinés à exciter l'ardeur des cultivateurs au moment des grands travaux collectifs.

11 Mais il y a cependant un cas de figure que nous n'avons pas encore envisagé, ni sur le plan des généralités, ni à propos de la société dioula ; c'est celui où le genre littéraire est désigné par référence à un groupe social, dont l'état peut être spécifié par différents types de critères : sexe (littérature féminine), âge (contes pour enfants, livres pour la jeunesse), profession ou activité (chants de paysans, de chasseurs...), condition socioéconomique (chansons « ouvrières ", chansons de bohémiens).

Dans tous les cas, la référence sociologique peut désigner, soit le producteur patenté du genre (chansons de bohémiens), soit le consommateur seul (contes pour enfants), soit c'est le cas le plus fréquent - les deux protagonistes de la communication littéraire : la littérature « féminine » ou la littérature " populaire » suggère qu'il s'agit de littératures faites par les femmes pour les femmes, ou par le peuple pour le peuple. Dans les conditions de production naturelles du folklore, il en sera souvent de même pour les récits "de marins", " de chasseurs ", produits et consommés à l'intérieur de la même catégorie sociale.

13 Il importe cependant de distinguer deux types de références à l'intérieur de cet ensemble.

Il y a le cas de celles qui caractérisent la catégorie sociale par rapport à une activité propre, ce qui, en principe, rattache d'emblée le genre à une thématique en relation directe avec cette activité. Nous sommes ainsi en quelque sorte renvoyés à un cas de figure déjà envisagé à propos des références circonstancielles : les chants de paysans, de marins, de chasseurs ne sont souvent autres que des chants agricoles, de marine, de chasse. Dans les premiers, on sait qu'il sera question de la vie champêtre, dans les seconds de ports, de bateaux et de tempêtes, dans les derniers, de gibier, de pistes et de traques. À Kong, on trouve d'ailleurs des dàndaga dònkili (chants de chasseurs) qui correspondent bien à ce type de situation, dans la mesure où ils sont exclusivement centrés sur la thématique de la chasse. La référence à un groupe social n'est plus ici qu'un substitut paradigmatique à la désignation de l'activité, et les deux possibilités fonctionnent souvent en distribution complémentaire.

Le problème est un peu différent lorsque le groupe social auquel il est fait référence n'est pas spécifié par une activité bien définie. La thématique est alors beaucoup moins explicite. Des expressions comme littérature "féminine ", littérature "populaire ", voire littérature "ouvrière » (car à la différence des cas précédents, il n'y est pas forcément question d'usines) ne sont véritablement fonctionnelles, au plan sémantique, qu'à partir du moment où il existe un minimum de complicité idéologique entre les interlocuteurs qui les emploient. Les termes "féminine", "populaire" ne leur apparaitront comme des qualificatifs pertinents pour caractériser un genre de littérature, que s'ils ont par ailleurs et a priori une certaine idée des modes d'expression, aux plans stylistique et thématique, de la femme, ou de ceux qui appartiennent aux couches dites «populaires ». Dans de telles expressions, il y a donc toujours une référence, au moins implicite, aux stéréotypes culturels et à l'idéologie.

On se trouve en présence d'un cas tout à fait semblable avec un genre de littérature dioula qui est baptisée wóloso dònkili (le déterminant wóloso désignant en l'occurrence un groupe social bien connu dans toute la communauté manding, à laquelle appartiennent les Dioula, celui des "captifs de case», c'est-à-dire des esclaves 
domestiques, qui sont «attachés » à une famille particulière). On pourrait penser qu'avec les wóloso dònkili, on retrouve une situation finalement assez proche de celle dans laquelle les catégories sociales étaient définies par leur activité : folklore du chasseur, du forgeron, du marin... Mais cela ne serait effectivement le cas que si la thématique dominante des chants de captifs était la condition d'esclave, ou les circonstances par lesquelles ils le sont devenus.

Or, si cela peut arriver, comme avec le chant suivant, dont on voit bien qu'il est pour les wóloso l'occasion d'affirmer leur identité comme groupe social :

síya ó síya $a^{3}$ kó án yé nyj̀gon bèn síya rá

quelle que soit (notre) race, (nous disons) que nous avons en commun une espèce,

jòn mùso $0^{4}$ dén,

(nous) fils de la femme esclave,

kó án yé nyògon bèn síya rá

voilà l'espèce que nous disons avoir en commun

18 Le fait reste néanmoins très exceptionnel, et tout à fait marginal par rapport au répertoire habituel de ces chants dont la principale caractéristique est la parodie obscène.

19 Nous comprendrons mieux cette fonction, si nous examinons de plus près les conditions d'énonciation des wóloso dònkili. On peut en effet se demander, comme nous l'avons fait à propos de nos autres exemples, si l'expression utilisée pour qualifier un tel genre signifie que les wóloso en sont les producteurs, les consommateurs ou les deux à la fois. Il faut donc savoir que les wóloso dònkili sont ainsi baptisés parce que ce sont les captifs, hommes et femmes (avec, comme nous allons le voir, un répertoire quelque peu différent selon les sexes) qui ont l'apanage exclusif de leur production. Mais ce n'est pas dans le même groupe social que se trouvent les destinataires, et les wóloso dònkili sont toujours adressés aux hóron, c'est-à-dire aux hommes libres, Dioula d'origine manding, ou qui se sont trouvés assimilés par d'autres voies que l'esclavage.

Pour bien comprendre ce que signifie cette relation entre wóloso et hóron, il nous faut rappeler quelques généralités sur la société manding dont les Dioula représentent une des composantes. Elle est en général divisée en trois grands groupes distincts : celui des hóron, nobles ou hommes libres, celui des nyàmakala qui regroupe un certain nombre de gens appartenant à des familles traditionnellement chargées, d'une part, d'activités artisanales spécifiques (travail du cuir, du fer...), d'autre part, de fonctions culturelles propres, généralement liées à l'exercice de la parole (c'est chez les nyàmakala que se recrutent les griots - jèli -), et enfin celui des jòn ou des wóloso, les esclaves, situés dans la hiérarchie traditionnelle au bas de l'échelle sociale. Soumis autrefois à un strict régime endogamique, qui, sans avoir totalement disparu, tend aujourd'hui à s'assouplir considérablement, ces groupes fonctionnaient comme des sortes de castes, système qui leur permettait de se perpétuer parallèlement les uns aux autres de façon relativement étanche.

21 À Kong, à la différence de la plupart des autres sociétés manding, la caste des nyàmakala n'existe pas en tant que catégorie spécifique, et le système se trouve simplifié par l'opposition des deux seuls groupes hóron et wóloso. Le statut de ces derniers correspondait, il n'y a pas si longtemps, à une condition socio-économique précise, dans la mesure où ils appartenaient à des familles de hóron qui les avaient acquis, soit par prise de guerre, soit par achat à d'autres communautés dont ils étaient devenus les prisonniers. Dans le monde manding, il y a eu jusqu'à la fin du XIXe siècle d'importants 
marchés d'esclaves, parmi lesquels d'ailleurs Kong, avec Sikasso et Bobodioulasso, figurait en bonne place. Les wóloso devaient donc servir leurs propriétaires : les femmes dans le cadre des activités domestiques, les hommes dans celui des activités économiques (surtout agricoles), mais en revanche ces derniers leur assuraient subsistance et protection.

La condition des wóloso n'est plus la même aujourd'hui, et même s'ils sont toujours considérés comme "liés » à des familles spécifiques de hóron, il s'agit là plus d'une sorte d'apparentement que d'une véritable relation de sujétion. Ils ont donc une condition à peu près équivalente à celle des hommes libres, et être wóloso n'a plus à l'heure actuelle d'incidence majeure du point de vue socio-économique. Il est intéressant de remarquer qu'on continue cependant à distinguer les hóron des wóloso, même si, l'endogamie des castes n'étant plus aussi stricte, cette distinction tend à devenir moins tranchée. Cela tient sans doute au fait que les wóloso ont eu très tôt, en même temps que des tâches spécifiques dans l'ordre économique, un rôle particulier dans l'ordre culturel, tout spécialement dans le domaine de l'expression verbale. Et c'est précisément à ces fonctions que nous allons nous intéresser maintenant.

À la plupart des manifestations publiques un peu importantes de la culture orale dioula, les wóloso sont présents et actifs, dans la mesure où ils reprennent sous une forme parodique obscène les genres « officiels » interprétés à cette occasion. La nature de leur participation est soumise à certaines règles qui dépendent étroitement de la nature des genres qui font l'objet de la contrefaçon. Selon que ceux-ci sont, d'après les lois de l'étiquette, produits par les femmes ou par les hommes, ce sont des wóloso de l'un ou l'autre sexe qui interviennent. Si le genre relève d'une production mixte, il en sera de même de l'intervention wóloso. Les parodies portent surtout sur des œuvres festives (tels les chants de danse, dòn dònkili qui forment une sorte de classe dans la nomenclature dioula) et cérémonielles (kónyon dònkili, chants de mariage; dénsagali

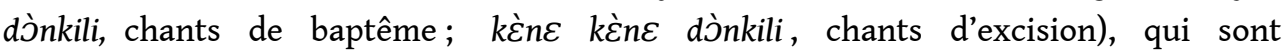
essentiellement le fait des femmes. La participation des femmes wóloso est de ce fait supérieure à celle des hommes. Ceux-ci interviennent surtout pour contrefaire les lásiri dònkili (devises chantées destinées à honorer une famille par l'évocation, souvent énigmatique, des hauts faits de ses ancêtres) et les kàlanjigi dònkili (chants interprétés par les jeunes garçons pour célébrer la fin de leurs études coraniques). Il leur arrive aussi de donner des chants qui ne sont plus à proprement parler des parodies, dans certaines conditions spécifiques sur lesquelles nous reviendrons tout à l'heure.

Ajoutons aussi que les autres sortes de limitation que sont susceptibles de connaître les genres « sérieux » qui font l'objet de ces caricatures burlesques (ils sont généralement restreints à un quartier ou à un groupe de quartiers, à une famille dans laquelle se déroule la cérémonie) touchent aussi l'intervention des wóloso. Ce sont ceux des familles ou des quartiers concernés qui se chargent de l'imitation grotesque. Par conséquent, on peut dire que les lois qui régissent l'énonciation de leurs parodies sont à peu près symétriques. D'ailleurs ces parodies se présentent sous une forme qui rappelle beaucoup celle de leurs modèles: mêmes structures linguistiques, mêmes airs de chansons. La seule différence tient généralement au changement de quelques mots dans l'énoncé qui lui donnent un sens obscène, et du point de vue de l'énonciation, au fait qu'elles sont l'apanage exclusif des wóloso qui jouent en quelque sorte le rôle de miroir déformant. 
Les choses se passent généralement selon des conventions très réglées. Les wóloso arrivent peu de temps après le début de la cérémonie et se mettent à reprendre quelques-unes des œuvres avec un certain nombre de variantes qui les rendent obscènes. Pendant quelque temps, les hóron présents feignent de les ignorer. Ensuite, ils manifestent-très théâtralement - qu'ils sont choqués, et ils expriment leur mécontentement aux wóloso en leur enjoignant de partir, mais ceux-ci continuent leurs parodies. Le même jeu se reproduit plusieurs fois jusqu'à ce que le hóron responsable de la cour où se déroule la fête ou cérémonie leur donne de l'argent pour «sauver l'honneur » des participants et leur « éviter la honte » d'entendre de pareilles horreurs. Alors les wóloso consentent à s'arrêter, ce qui ne les empêche pas de rester à la séance, et même le plus souvent de continuer à participer aux chants produits, mais cette fois sans intention parodique.

Nous allons présenter quelques-uns de ces chants de captifs, en les rapportant, chaque fois que cela sera nécessaire, à l'œuvre originale qu'ils ont déformée. Nous pourrons ensuite nous interroger sur la signification sociale profonde de telles pratiques.

Intéressons-nous d'abord aux wóloso dònkili qui parodient les chants de danse (dòn dònkili).

Wóloso dònkili $\mathrm{n}^{\circ} 1$

1

Ala ánnori ní sàwaba bèn ó

a Que Dieu nous fasse rencontrer une grande joie, $\hat{o}$ bis béma $a^{5}$ Basirifé tá lè hóronma

Par la grâce du vieux Basirifé.

Ala ánnori ní sàwaba bèn ó

b Que Dieu nous fasse rencontrer une grande joie, ô

à dógomuso Nahusata tá lè hóronma

Par la grâce de sa petite sœur Nahusata.

Ala ánnori ní sàwaba bèn ó

c Que Dieu nous fasse rencontrer une grande joie, ô

à dógomuso Naminata tá lè hóronma

Par la grâce de sa petite sœur Naminata.

Ala ánnori ní sàwaba bèn ó

Que Dieu nous fasse rencontrer une grande joie, $\hat{o}$

d é béma Basirife lè hóronma

Eh, par la grâce du vieux Basirifé.

Ala ánnori ní sàwaba bèn ó

Que Dieu nous fasse rencontrer une grande joie, $\hat{o}$.

2

à kó wúlu yé à lá á á byc̀ yé à kàw

e On dit que la queue chante ça, ah! Le con ${ }^{6}$ le reprend (après elle),

jùla wúlu yé à lá byغ̀ yé à kàw

La queue du Dioula chante ça, le con le reprend après elle.

é é wúlu yá bón wúlu yé

f Hé, une queue peut être plus grosse qu'une autre queue, ter wúlu mán dí wúlu yé

(mais) une queue n'est pas plus agréable qu'une autre queue.

jùla wúlu lòko yé yélengenden

g La façon de s'arrêter de la queue de Dioula, c'est (de faire) yelengenden,

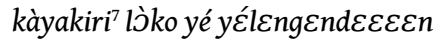

La façon de s'arrêter du sexe masculin c'est de faire yelengendeeeen ${ }^{8}$.

Ce premier chant a été dit par des femmes à l'occasion d'une danse de gbòbi, mais des hommes auraient pu s'y associer, puisqu'à la différence de certaines autres danses, le 
gbòbi admet la mixité pour la production des chants. Toutefois, il pourrait être aussi bien une parodie d'autres dòn dònkili (kúrubi, sábe, yàgba...) car, même s'il existe un répertoire de chants plus ou moins spécifique à chaque danse, il y a néanmoins une partie commune. C'est le cas notamment des chants qui servent à introduire la séance, et qui constituent un répertoire commun à toutes les danses.

Dans tous les cas, ces chants d'introduction comprennent des sortes de prières: bénédictions et louanges adressées à Dieu. C'est une façon de se concilier sa bienveillance au début des réjouissances. À ces chants à caractère religieux, se combinent parfois les salutations ou les louanges des hauts personnages de la cour ou du quartier où a lieu la danse. Ces salutations adressées à une personne ou à une famille apparaîtront tout particulièrement lorsque, comme c'est possible pour un certain nombre de danses, la fête aura été commandée par quelqu'un pour célébrer un événement heureux. Celui-ci donne alors de l'argent aux gens du quartier qui organisent la danse pour les récompenser de leur prestation. En revanche, l'usage veut que les interprètes mettent à l'honneur cette personne et sa famille dans leurs chants d'introduction. Parfois ces invocations à Dieu et à la personne qui a commandé la danse peuvent se combiner dans une même chanson, comme c'est le cas pour le chant de gbòbi qui se trouve parodié par ce premier wóloso dònkili.

Généralement, après ces invocations préliminaires, arrivent dans la danse des chants formés d'énoncés sentencieux, courants dans la culture. En fait, des proverbes chantés ressurgiront périodiquement tout au long de la prestation, mais il est fréquent qu'on en trouve une série au début, afin de "chauffer " la séance, car ils sont bien connus de tous. Puis viennent ensuite des chants plus divers faisant allusion à des événements liés à l'histoire des quartiers qui produisent la danse, ou à des expériences relatives à la vie culturelle des Dioula.

Le wóloso dònkili que nous venons de présenter est en fait un amalgame de ces différents types de chants d'introduction. On peut remarquer qu'il se divise en deux grandes parties. Une première qui n'a rien de parodique du point de vue textuel ${ }^{9}$ et qui se contente de reprendre fidèlement les invocations du chant de danse telles qu'elles ont été énoncées. Celle-ci comprend plusieurs strophes de même type, du fait que les invocations successives, en même temps qu'à Dieu, s'adressent à plusieurs personnes différentes de la famille de celui qui a commandé la danse : le vieux Basirifé. La seconde partie, elle, en introduisant la thématique proprement paillarde fait basculer l'œuvre dans la production wóloso. La première strophe parodique (e) est en fait une sorte de conclusion burlesque à toutes les invocations précédentes, qui, a posteriori, en modifie complètement l'intention, en les rendant brusquement dérisoires; nous y apprenons, en effet, que les énonciateurs des prières et bénédictions, reprises mot à mot du chant de gbòbi, sont le pénis et le vagin.

La strophe (f) quant à elle, est la déformation d'un lámara (proverbe) très connu, qui, comme les autres lámara, apparaît couramment dans la thématique des chants de danse :

kógo ká bòn kógo yé,

kógo mán dí kógo yé,

ce qui veut dire littéralement « une variété de sel peut avoir des grains plus gros qu'une autre, elle n'est pas plus agréable pour autant ", ce qu'il faut comprendre de la façon suivante : une variété de sel peut être plus grosse qu'une autre, ce n'est pas pour autant qu'elle salera mieux. Ce proverbe est employé pour signifier qu'il ne faut pas toujours 
se fier aux apparences d'une chose ou d'une personne pour juger de son efficacité. La parodie wóloso a décalqué exactement la matrice originale en se contentant de remplacer kógo (sel) par wúlu (sexe masculin), ce qui d'ailleurs permet au proverbe de conserver le même sens avec une autre image renvoyant à une thématique grivoise d'une crudité affirmée qui s'accentue encore avec la dernière strophe (g).

Nous allons présenter maintenant deux wóloso dònkili, toujours chantés par des femmes qui parodient un type de chant de danse très classique, qu'on retrouve principalement dans le kúrubi, mais aussi dans des danses telles que le gbòbi ou le sábe. Ces chants consistent à peindre le portrait d'une belle jeune fille, selon les canons dioula de la.beauté féminine. En voici un prototype, produit comme kúrubi dònkili, dans sa traduction française :

Hé ! Le marabout ${ }^{10}$ nous attire, ô.

Au bord du fleuve, le marabout nous attire, ô, au bord du fleuve.

Et tu marches en regardant ton ombre, et en nous attirant, $\hat{o}$, au bord du fleuve.

Et tu marches en te déhanchant et en nous attirant, ô, au bord du fleuve (...)

L'œil comme une étoile nous attire, ô.

La chevelure comme une queue de cheval nous attire, $\hat{o}$, au bord du fleuve.

Celle qui a la taille comme le lion nous attire, ô.

Et tu marches en regardant ton ombre et nous attirant, ô, au bord du fleuve (... $)^{11}$

Et voici deux wóloso dònkili qui sont une parodie, non de ce texte particulier, mais de chants qui relèvent de cet archétype.

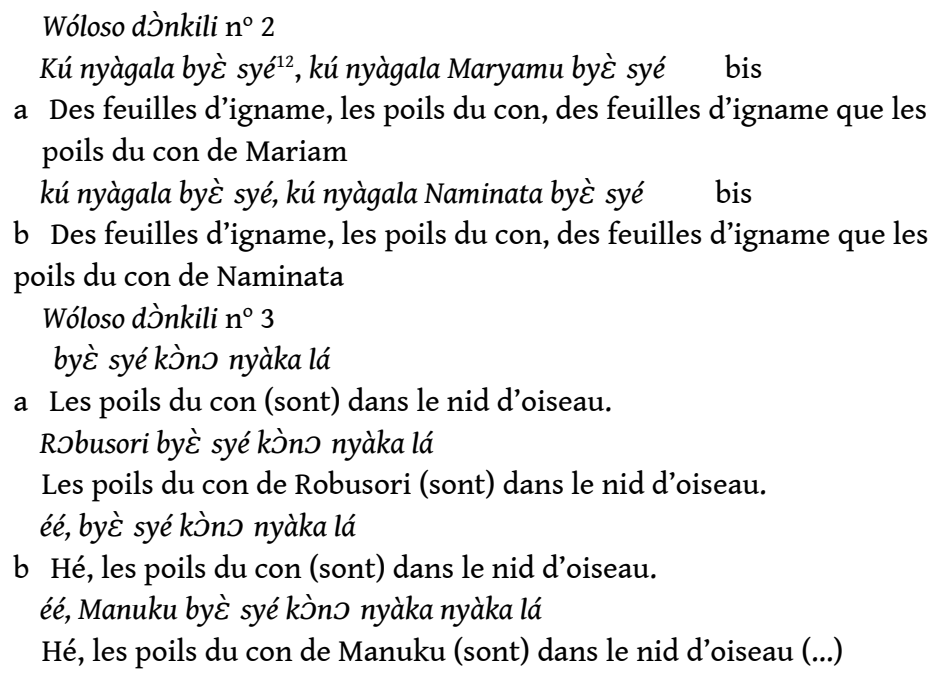

L'énumération continue encore ainsi longtemps, mais il est inutile de la prolonger, puisque les phrases restent identiques, seul le nom de la personne change.

Dans la plupart des parodies de ce type, ce sont les poils du pubis qui sont le substitut dérisoire des traits physiques dont on loue les mérites dans la véritable chanson. $\mathrm{Au}$ reste, la parodie repose sur le même principe que son modèle et, comme lui, utilise le principe de la métaphore valorisante, mais sur un mode burlesque. De même que, dans le chant de kúrubi, les yeux de la jeune fille sont comme des étoiles et sa chevelure comme une queue de cheval, de même, dans le chant de wóloso, les poils du pubis sont comme des feuilles d'igname ${ }^{13}$, ou sont comparés aux duvets et végétaux qui tapissent les nids d'oiseau.

Voici une dernière parodie de dòn dònkili donnée à l'occasion de la Tabaski ${ }^{14}$. Cette fête donne lieu à la sortie de très nombreuses danses. À cette occasion, les femmes wóloso se promènent dans chaque quartier, de cour en cour, en brandissant les testicules des 
moutons égorgés, et en chantant des chansons qui reprennent de façon caricaturale le style des dòn dònkili qui ont cours à ce moment-là.

Wóloso dònkili $\mathrm{n}^{\circ} 4$

byغ̀ wé byغ̀ byé si sàraman té bis

a Le con, ô, rien n'est plus agréable que ça, byغ̀ yé gbànfula bó kàramogo yé

b Tout le monde retire sa coiffure pour le maitre coranique, kàramogo yé gbànfula bó byغ̀ yé

c Le maître coranique retire sa coiffure pour le con.

Le kàramogo, ou maitre coranique, est souvent la cible des chants de wóloso, notamment au moment de la cérémonie du kàlanjigi par laquelle les élèves célèbrent la fin de leurs études coraniques, et où il est d'usage de mettre son mentor à l'honneur. Ce chant d'ailleurs aurait très bien pu apparaître à l'occasion d'un kàlanjigi, mais il aurait alors été interprété par des hommes. Le kàramogo est aussi un personnage important de la Tabaski, grande fête musulmane, ce qui explique qu'il inspire une bonne partie du répertoire wóloso donné à cette occasion.

Ici, tout l'intérêt du chant, en dioula, provient de la paronomase qui, d'abord à l'intérieur du premier verset, mais surtout entre le deuxième et le troisième verset, crée une sorte de jeu de mots entre byغ̀ dénomination vulgaire du sexe féminin, et byé signifiant "tous » (ou « rien » sous la forme négative byé sí, au verset 1). La parenté phonétique de ces deux paronymes, confinant à l'homonymie, puisque seul le ton change, permet un effet de chiasme au plan du signifiant (le verset 2 et le verset 3 sont construits selon une symétrie inverse presque parfaite, au ton de bye près). Mais le chiasme du signifiant n'est pas suivi par un chiasme équivalent au niveau du signifié (on attendrait normalement: "tout le monde retire sa coiffure pour le maitre coranique, le maître coranique retire sa coiffure pour tout le monde»), et le

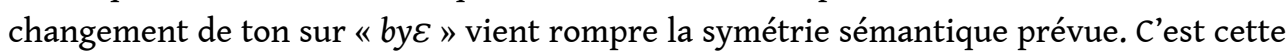
tension entre les deux plans, celui du signifiant et celui du signifié, qui alimente dans ce texte le jeu poétique, sur le mode du calembour.

41 Nous allons maintenant donner un échantillon de wóloso dònkili créés comme parodies de chants cérémoniels, en continuant pour l'instant de puiser nos illustrations dans le répertoire des femmes.

Wóloso dònkili n ${ }^{\circ} 5$

éé ń yé dùgu tálen ó

a Hé, j'avais passé la moitié de la nuit ${ }^{15}$, ô

ní mògonaden kà wóro

b Quand l'enfant d'une femme est né.

jó lé yéa dénbaya ké ó óó

c Mais qui donc l'a engendré ?

kónyumanlonbari ${ }^{16}$ f́́re

d Regarde cet ignorant du bien,

éle ná tì kí éle wóro

e C'est ta mère qui t'a mis au monde, jón nì kà í dénbaya ké óóó

$f$ (Mais) qui donc t'a engendré ?

Ce chant est une parodie de chant de baptême (dénsagali dònkili). Au cours de cette cérémonie, l'usage veut que les chants qui l'accompagnent rappellent, entre autres choses, la généalogie de l'enfant, notamment du côté de la lignée paternelle, puisque la filiation est patrilinéaire chez les Dioula. Le chant du captif, moins obscène ici qu'irrespectueux, prend le contre-pied de cette pratique puisqu'il considère l'enfant 
comme un bâtard, en feignant d'ignorer son ascendance paternelle, ce qui, dans toute autre circonstance, serait considéré comme une injure très grave.

Les quatre autres chants qui vont suivre sont des parodies de chants de mariage. Le premier est la contrefaçon d'un kónyon nàngbe dònkili (chants de mariage du nàngbe) type d'œuvre qui apparait lors d'un rite qui a lieu la veille de la nuit de noces, ce qui explique la thématique rencontrée :

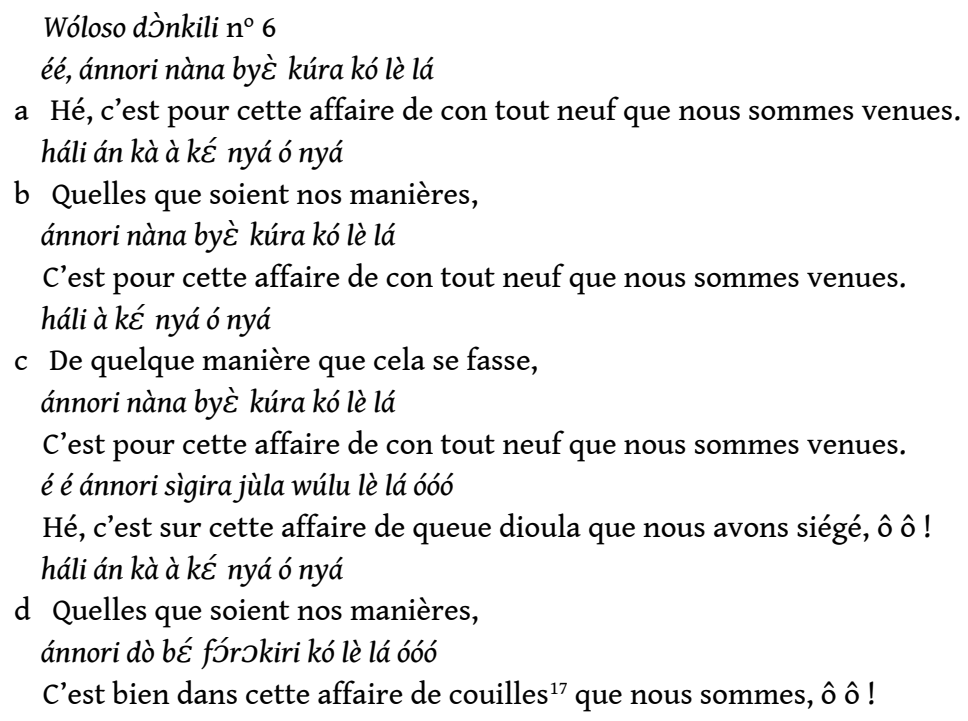

On peut constater que le chant n'est fait que de deux phrases de base qu'on reprend en y brodant de très légères variantes. C'est le principe classique de presque tous les chants dioula. Ici, ces variantes permettent d'enrichir la thématique paillarde en multipliant les références aux organes sexuels féminins et masculins. La phrase qui commence chacune des trois strophes principales $(b, c, d)$, qui connaît elle-même une légère variante, est une allusion au comportement des wóloso à qui on reproche toujours leur vulgarité, en leur enjoignant de déguerpir.

Les trois chants suivants, toujours liés à la cérémonie du mariage, sont des contrefaçons de kónyon sùsu dònkili (chants de mariage où l'on pile) donnés les lundi, mardi, mercredi de la première semaine. Un grand mortier a été enfoncé sur le gbéndege ${ }^{18}$ de la cour de la mariée, et les femmes du quartier, aidées par les vieilles d'autres quartiers, pilent à tour de rôle du maïs, du mil, du sorgho, afin de préparer la nourriture qu'on servira pour les festivités. Lorsqu'une équipe de femmes, généralement apparentées, se met à piler, les autres, pour piquer leur amour propre, chantent alors certaines sentences, parmi lesquelles se trouvent les lásiri dònkili attachés à leur nom, ces chants dits de généalogie qui honorent les familles, en évoquant les hauts faits de tel ou tel de leurs ancêtres prestigieux ${ }^{19}$.

Wóloso dònkili $\mathrm{n}^{\circ} 7$

é é wúlu wé wé, wúlu wé wé

a Hé, la queue, tralala, la queue, lala, wúlu dò ma bóduga lón

La queue ne connaît même pas l'endroit d'où elle sort, jùla wúlu bora byc̀ rá

La queue du Dioula est sortie du con.

é é wúlu wé, wúlu wé

b Hé, la queue, lala, la queue, lala,

ń kó à dò má bóduga lon

Je dis qu'elle ne connaît même pas l'endroit d'où elle sort, 
jùla wúlu bóra byc̀ rá

La queue du Dioula est sortie du con,

ń kó à dò má bóduga tó óóó

Je dis qu'elle ne déclare même pas l'endroit d'où elle sort, ô, ô, ô.

Puisque la fonction valorisante des lásiri dònkili tient à l'énonciation de l'ascendance prestigieuse dont peuvent se targuer les hóron, il s'agit tout d'abord pour les wóloso de tourner en dérision cette prétention, en s'en moquant. C'est un peu comme s'ils disaient aux hóron, lorsque ceux-ci se vantent de sortir d'une grande famille : «Et ta queue, tu sais d'où elle sort ? Elle sort du con ». Comme pour la contrefaçon du chant de baptême, celle-ci laisse donc sourdre une certaine rivalité entre les deux groupes.

te ce chant tient encore à un jeu de mots, qui se perçoit aussi dans la traduction française, et qui repose sur le double sens, propre et figuré, du verbe bó (sortir). Celui-ci signifie à la fois, au figuré, " provenir de ", «tirer son origine », sens qu'il prend dans la première proposition (« la queue ne connaît même pas l'endroit d'où elle sort»), et, au propre, «s'extraire d'un lieu», sens de la proposition suivante («la queue est sortie du con »). On est là devant un procédé de glissement de sens du même genre que celui qu'on rencontre dans la boutade populaire en France: «l'homme descend du singe, et le singe descend de l'arbre ».

Wóloso dònkili $\mathrm{n}^{\circ} 8$

i yé Sangare wéle, Ture dén

Appelle Sangaré, l'enfant des Touré,

díya yé yàn óó

Le bonheur est ici, ô ô,

é kàya dánna île míin kòsòn

a Hé ! C'est grâce à toi que la verge ${ }^{20}$ a été créée,

é kàyakiri dánna ile mín kòsòn

Hé ! C'est grâce à toi que les parties ont été créées,

éjùla wúlu dánna ile mín kòsòn

Hé ! C'est grâce à toi que la queue de Dioula a été créée,

í Sangare wéle, Ture dén

Appelle Sangaré, l'enfant des Touré,

díya yé yàn óó

Le bonheur est ici ô ô,

é kàya dánna île mín kòsòn

Hé ! C'est grâce à toi que la verge a été créée,

b é kàyakiri dánna ile mín tógo rá

Hé ! C'est en ton nom que les parties ont été créées,

é jùla wúlu dánna ile mín tógo rá

Hé ! C'est en ton nom que la queue de Dioula a été créée,

i yé Sangare wéle, Ture dén

Appelle Sangaré, l'enfant des Touré,

díya yé yàn ó

Le bonheur est ici, $\hat{o}$.

On retrouve toujours le principe d'une structure binaire avec quelques variantes. À la différence du précédent, qui prenait le contrepied des lásiri dònkili, en mettant en scène une sorte de personnage burlesque, «wúlu» (la queue) qui ignorait son origine, ce wóloso dònkili est beaucoup plus un décalque fidèle de l'archétype original. Si on le compare avec le lásiri dònkili cité en traduction française dans la note 19, on constate qu'il obéit exactement aux mêmes principes : invocation du nom de l'ancêtre, évocation des exploits ou bienfaits qu'on peut lui attribuer. La seule différence évidemment est 
que, dans la parodie, ces prétendus mérites sont parfaitement grotesques : on attribue à l'ancêtre la création du pénis et des testicules. On notera une progression dans le texte, allant de termes relativement neutres pour renvoyer au sexe masculin (kàya, kàyakiri) à des termes plus crus (wúlu).

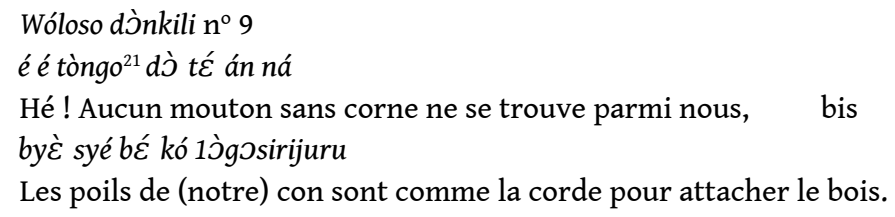

Pour bien comprendre ce dernier énoncé, il faut savoir que l'image du bélier sans corne est employée dans nombre d'expressions idiomatiques dioula pour désigner une personne faible, sans défense. Il y a même un proverbe très connu qui dit: «sàgajigi tòngo jùsu bé í rá, gbàn kó lè tóra », ce qu'on pourrait traduire par : "bélier sans corne, peut-être que tu as du cœur ${ }^{22}$, reste quand même le problème des cornes ».

Ce chant, produit à la même occasion que les deux précédents, c'est-à-dire au moment du rite de kónyon sùsu, fonctionne selon un principe un peu différent. Il s'agit moins d'une parodie que d'un substitut de lásiri dònkili. Les wóloso qui, par définition, ne peuvent bénéficier de lásiri dònkili propres, puisqu'ils n'ont pas d'ascendance reconnue dans la société dioula, se sont créé, à leur manière, leurs chants de louange particuliers pour les remplacer. La première personne du pluriel qu'on trouve dans le texte, "án » (nous), renvoie donc à la communauté wóloso.

51 Le chant a été proféré par des femmes wóloso, alors que c'étaient d'autres femmes wóloso qui étaient en train de piler. Comme il est d'usage, au cours du rite du kónyon sùsu, d'exciter l'ardeur de celles qui passent à tour de rôle au mortier ${ }^{23}$ en leur chantant des chants d'encouragement (où se mêlent les lásiri dònkili attachés à leur famille), et qu'en l'occurrence elles ne pouvaient le faire, elles ont eu recours à ce succédané. On comprend bien la signification du chant: il n'y a pas de mauviette parmi nous. Contrairement à celle du bélier sans corne, l'image des poils du pubis (que nous avions déjà rencontrée dans les chants $\mathrm{n}^{\circ} 2$ et no 3 ), qui sont solides comme de la corde à lier les fagots, évoque naturellement l'idée de la force. Mais une fois de plus, évidemment, du fait du burlesque du trait physique de référence, la louange apparaît dérisoire ; et, dans la mesure où, cette fois, cette dérision s'exerce moins à l'encontre des hóron - qui dans cet acte de communication particulier deviennent plutôt témoins que destinataires du message - qu'à l'encontre des wóloso eux-mêmes, on peut considérer qu'on est passé de l'irrévérence ironique à l'humour.

Toutefois, il faut savoir que, d'après les informations qui nous ont été données, un chant de ce type peut être utilisé de façon moins humoristique, dans le cas d'un différend entre hóron et wóloso. Il exprime alors un véritable défi.

Nous allons terminer la présentation de notre échantillon par quelques chants d'hommes, choisis comme représentatifs de leur répertoire. Parmi ceux-ci, il y a tout d'abord les parodies de kàlanjigi dònkili, ces petits chants donnés par les élèves des kàramogo lorsqu'ils quittent l'école coranique :

Wóloso dònkili $\mathrm{n}^{\circ} 10$

éé ń kàramogo Balaji yéán kàlan lá mín

a Hé, au moment où mon maître Balaji nous enseignait,

ò sága lánin Balaji jùla wúlu yá dí

À ce moment-là, la queue dioula de Balaji était bonne. 
$m \grave{\varepsilon} n m \dot{\varepsilon} n m \dot{\varepsilon} n m \dot{\varepsilon} n m \dot{\varepsilon} n$

men men men men men ${ }^{24}$

é é à dj̀goce Nfasirima yé án kàlan lá mín

b Hé, au moment où son petit frère Nsafirima nous enseignait, ò sága lánin Nfasirima jùla wúlu yá dí

À ce moment-là, la queue dioula de Nsafirima était bonne.

$m \dot{\varepsilon} n m \dot{\varepsilon} n m \dot{\varepsilon} n m \dot{\varepsilon} n m \dot{\varepsilon} n$

men men men men men

él háji Tijani yé án kàlan lá mín

c Au moment où El Hadj ${ }^{25}$ Tidyani nous enseignait, ò sága lánin, Tijani jùla wúlu yá dí

À ce moment-là, la queue dioula de Tidyani était bonne.

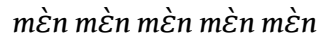

men men men men men

é é ń bilance ${ }^{26}$ Bamoriba yé án kàlan lá mín

$\mathrm{d}$ Hé, au moment où mon beau-père Bamoriba nous enseignait, ò sága lánin, Bamoriba wúlu yá dí

À ce moment-là, la queue de Bamoriba était bonne.

$m \varepsilon \grave{n} m \dot{\varepsilon} n$ mèn $m \varepsilon \grave{n} n m \dot{\varepsilon} n$

men men men men men

à dògoce Batibu yé án kàlan lá mín

$\mathrm{e}$ Au moment où son petit frère Batibu nous enseignait,

ò sága lánin Batibu júla wúlu yá dí.

À ce moment-là, la queue dioula de Batibu était bonne.

$m \dot{\varepsilon} n m \dot{\varepsilon} n m \dot{\varepsilon} n m \grave{\varepsilon} n m \dot{\varepsilon} n$

men men men men men

Ce chant est un décalque très fidèle du kàlanjigi dònkili qui lui a servi de modèle puisqu'il reprend exactement le texte à un mot près. La phrase originale du chant était :

ń kàramogo Balaji yé án kàlan lá mín

Au moment où mon maître Balaji nous enseignait,

ò sága lánin Balaji kúma yá dí

À ce moment-là, la parole de Balaji était bonne.

Dans la contrefaçon, seul a changé le mot «kúma», remplacé par «wúlu». Il faut signaler aussi l'ajout, à la fin de chaque couplet, de l'onomatopée obscène «mغ̀n mèn $m \varepsilon \grave{n} . .$. » qui suggère, d'après nos informateurs, que l'énonciateur du texte parodique est le vagin. Dans la plupart des cultures, le sacré a toujours été une cible de choix pour la parodie irrévérencieuse, et le folklore obscène français pourrait donner ainsi de nombreux exemples de contrefaçons facétieuses de réponses au catéchisme, ou de cantiques, aussi bien en latin qu'en français.

Nous allons maintenant présenter trois cas un peu particuliers, puisqu'il s'agit de wóloso dònkili qui, s'ils restent faits sur le modèle général du chant dioula (une ou deux phrases assez courtes, reprises avec quelques variantes), ne sont pas à proprement parler la parodie d'un chant spécifique, répertorié dans le patrimoine folklorique autochtone.

Wóloso dònkili $\mathrm{n}^{\circ} 11$

ń béma wé ń béma wé

Mon grand-père, hé, mon grand-père, hé !

byc̀ sòngon dí

Donne le prix du con,

í kà byè mín kè sú rá

De ce con que tu as eu cette nuit, 
byغ̀ sòngon dí

Donne le prix du con. cérémonie, mais lorsqu'ils apprennent qu'un homme de caste hóron a pris pour amante passagère une femme wóloso. La chose se comprend d'autant mieux qu'à l'origine, les castes, comme nous l'avons dit, étaient endogamiques dans la société manding, et que ce faux pas représentait en quelque sorte une incursion en zone interdite, privauté que, comme partout où il y a eu de l'esclavage, le maître s'accordait sans doute volontiers. Il s'agissait pour les wóloso qui fournissaient ainsi - même involontairement - un objet à son plaisir, de réclamer un dédommagement. Aujourd'hui, la démarche a sans doute moins de sens, dans la mesure où il peut arriver assez couramment qu'un hóron épouse une femme wóloso - l'inverse est beaucoup plus rare ${ }^{27}$. Cependant, il est encore assez fréquent, si un hóron a des relations sexuelles extra-conjugales avec une captive, que les hommes wóloso du quartier, s'ils l'apprennent, viennent dans sa cour pour réclamer «le prix du sexe », par un chant de ce type, qu'ils répèteront aussi longtemps que le hóron ne se sera pas exécuté, en leur versant quelque argent.

Wóloso dònkili $\mathrm{n}^{\circ} 12$

éé béma kà dógoma dí

Hé ! Le vieux a donné un petit quelque chose,

án dò má ò dómu bán hálìò ní à być bis

Nous n'avons quant à nous pas encore fini de le manger avec tout le reste.

béma bwò kèra ń kàn

Le vieux a déposé sa crotte sur moi.

Les chants de ce type sont dits lorsque le hóron, qui a prié les wóloso de déguerpir à une cérémonie quelconque, s'est enfin décidé à leur donner un petit cadeau ; aujourd'hui il s'agit toujours d'un peu d'argent. Ce wóloso dònkili a été donné par des hommes, mais il aurait pu être aussi bien produit par des femmes. Il a pour fonction de remercier le donateur en faisant connaître publiquement qu'il a fait un cadeau, mais on voit qu'une fois de plus ces remerciements sont ambigus, car ils ne sont pas dénués d'une certaine ironie.

Le terme dógoma, « un petit peu », laisse supposer qu'il s'agit d'un petit cadeau (sousentendu : il aurait pu se montrer plus généreux), et le présent du donateur, au lieu d'être valorisé comme l'exige la politesse, selon le code des bonnes manières dioula, est assimilé à de la crotte, une insolence supplémentaire. En principe ce sont ces types de chants dont les wóloso se servent pour clore leurs séries parodiques, lorsqu'ils ont touché le fruit de leur prestation.

Wóloso dònkili $\mathrm{n}^{\circ} 13$

hóron má nyáni lón, bó síya yé ééé

L'homme libre n'a pas connu la misère, sors donc avec tes coutumes, eh! bis

hóron má nyáni lón, í tí bó síya tèn yé níń

L'homme libre n'a pas connu la misère, ne sors-tu pas avec tes coutumes?

La thématique de ce dernier chant rappelle un peu celle du tout premier que nous avons cité dans nos pages d'introduction. Certes, il peut à la rigueur fonctionner comme un pastiche de chant de danse, dans la mesure où un certain nombre d'entre eux sont ainsi faits d'aphorismes sur la condition humaine, du genre: "tous les hommes sont des esclaves de Dieu ", mais on voit qu'ici il ne s'agit pas de contrefaçon burlesque, et qu'il est presque abusif de parler alors de parodie. D'autre part, un chant de ce type est souvent produit sans fonctionner comme le pastiche d'un genre littéraire reconnu, mais à l'occasion d'un différend important entre hóron et wóloso, par lequel il 
exprime un véritable défi, signifiant à peu près, en langue familière : « celui qui n'a pas été esclave ne connaît rien à la souffrance - c'est-à-dire à la vie - même s'il la ramène avec ses coutumes ».

61 Nous arrêterons là la présentation de notre échantillon, qui rend compte des modèles les plus représentatifs du genre et donne une idée de l'ensemble de son répertoire. À partir de ce petit corpus, nous pouvons établir que ces chants ont deux caractéristiques nettement dominantes : l'obscénité et la parodie.

Ces deux traits prendront toute leur signification si on considère à la fois la nature des modèles parodiés, et celle des partenaires en cause dans l'acte de communication institutionnelle accompli au moyen des wóloso dònkili.

Les objets de la parodie, ce sont d'autres genres littéraires chantés, mais dont il faut remarquer que l'énonciation est toujours liée à d'importantes coutumes de la culture dioula. Leur signification sociale implique donc beaucoup plus que le simple contenu linguistique de leur énoncé. Ils sont produits à l'occasion de fêtes datées (souvent d'ailleurs en rapport avec l'islam, dans le cas des chants de danse) ou bien à l'occasion de cérémonies sociales (mariage, baptême...) ou religieuses (kàlanjigi : célébration de la fin des études coraniques). Leur mise en cause par la caricature parodique, est donc certes celle d'un discours et d'une idéologie, mais bien au-delà encore, la dérision de toute une culture à travers ses pratiques institutionnelles.

Or, qui sont les auteurs de cette dérision? Les captifs, c'est-à-dire ceux que leur condition désigne à la fois comme des marginaux par rapport à cette culture, puisqu'ils sont par définition d'origine étrangère (même très assimilés, ils sont probablement restés longtemps marqués en tant que "métèques»), et comme les dominés par excellence, puisque jusqu'au début du siècle, ils étaient véritablement " possédés » par des familles. De cette condition, une certaine condescendance un peu méprisante est demeurée à leur encontre de la part des hóron. Que ce soit eux les auteurs de telles parodies n'a rien de surprenant. Sous tous les cieux, ce sont toujours les opprimés, les faibles qui, au moyen des formes de culture populaire, verbales ou graphiques, font des caricatures irrévérencieuses des discours et des manières de leurs maitres, jamais l'inverse.

Ce qui est déjà plus rare et plus déconcertant, c'est qu'ils ne produisent pas ces parodies pour leur consommation interne, mais que justement ils viennent les chanter véritablement sous le nez de ceux qui sont les dépositaires de cette culture, et qui sont censés la prendre au sérieux. Il y a là, au moins en apparence, une sorte de défi dont l'audace a de quoi étonner. Nos enfants de chœur n'allaient pas chanter leurs parodies obscènes de cantiques devant Monsieur le Curé. Certes, il y a des précédents connus. Les fous du roi, amuseurs de Sa Majesté, ne ménageaient pas, dit-on, leur insolence à l'égard des puissants, ni parfois à l'égard du prince lui-même, et la parodie était pour eux un instrument de choix. Dans le monde manding même, le griot, qu'il ne faudrait certes pas confondre avec les wóloso, car il a un autre prestige, tout en ayant pour fonction de louer et d'honorer les puissants, peut se permettre à leur égard certaines privautés que nul autre ne pourrait s'autoriser. Mais dans le cas qui nous occupe, il s'agit moins d'individus au statut quelque peu exceptionnel que d'une classe tout entière. l'histoire, et il ne nous est pas possible de savoir aujourd'hui comment sont apparus les 
wóloso dònkili, ni comment s'est instaurée cette pratique qui consiste à les dire publiquement devant les hóron à l'occasion de cérémonies. On ne peut sur ce point que faire des suppositions. Probablement sont-ils nés d'une certaine agressivité de ces captifs à l'égard de la culture de leurs maîtres qui n'était pas la leur. En la tournant en ridicule, ce sont des hóron eux-mêmes dont ils se moquaient ; ils trouvaient sans doute là une sorte de revanche à leur condition peu enviable. Et l'on peut supposer qu'au départ ces chants ont surtout circulé à l'intérieur du groupe wóloso, comme une véritable " parole buissonnière ", avec toute la valeur subversive que cela implique.

Mais il est vraisemblable qu'au bout d'un certain temps, comme presque toujours en pareil cas, les hóron ont eu progressivement connaissance de ces parodies dont ils faisaient les frais. Et il n'est pas interdit de penser que dès lors, il a pu y avoir, plus ou moins consciemment sans doute, une certaine tentative de récupération de ce type de discours, en lui accordant un statut institutionnel. Le groupe dominant des hóron pouvait trouver à cela un double intérêt :

- d'une part, en encourageant les wóloso à chanter publiquement devant eux, ils favorisaient le défoulement de l'agressivité latente que ces derniers ne pouvaient manquer d'éprouver à leur encontre, et ils la contrôlaient plus facilement dans la mesure où ils en étaient les témoins. On sait bien que l'expression de l'agressivité par la parole permet souvent d'éviter qu'elle ne se traduise par d'autres actes, plus dangereux. Et il est sans doute plus confortable pour le destinataire que cette agressivité, même si elle reste verbale, s'exprime sur le mode de la dérision, par la thématique paillarde, que sur celui de la violence. Du même coup, ils accordaient ainsi aux captifs un certain statut culturel qui, bien que restant mineur, était susceptible de favoriser leur intégration sociale ;

- d'autre part, il faut se rappeler que les hóron, de par leur condition, sont tenus à certaines règles de bienséance. Ils doivent se conduire comme des gens respectables, et, l'islam favorisant une certaine forme de "puritanisme", ils soignent particulièrement leur distinction, dans leur vêture comme dans leurs manières. Ils affichent en public une pudeur presque excessive, et pour eux, selon l'étiquette, la plus grande réserve est de mise. Il y a toute une idéologie aristocratique du hóron, une sorte d'idéal de "l'honnête homme dioula ", à qui sa classe impose une certaine conduite et, bien entendu, il serait tout à fait impensable qu'il puisse proférer publiquement les obscénités des chansons de wóloso. Cela nous conduit à penser que le fait de faire dire à d'autres ce que sa condition lui interdit, peut être l'occasion de son propre défoulement. Dans une société où l'étiquette de la tradition est souvent contraignante, il est bien possible que ceux qui y sont soumis éprouvent inconsciemment le besoin de prendre un certain recul par rapport à ce pesant appareil qu'ils subissent eux-mêmes, bien que l'idéologie dominante les ait préparés à l'accepter. Comme ils ne peuvent prendre seuls ce recul, les wóloso sont là pour le faire à leur place, exprimant ainsi les phantasmes de leur propre refoulement, imposé par le code culturel.

Il ne s'agit certes là que d'une hypothèse. Mais il est clair, lorsqu'on a été un témoin direct des séances où apparaissent des wóloso dònkili que les hóron, même s'ils manifestent - avec une théâtralité souvent bien excessive pour être vraiment sincère la honte qu'ils éprouvent devant ces chants obscènes, prennent un très grand plaisir à entendre et à voir faire par d'autres ce que la bienséance leur interdit.

Le rôle social des wóloso, de par le contenu de leurs productions, aurait donc une double face : la face officielle et la face cachée ou inconsciente. Théoriquement, dans la mesure où ils provoquent la honte chez les destinataires de leurs chants, ils disposent d'une parole redoutée qui, par une sorte de chantage, leur procure un bénéfice économique, 
puisqu'on cherche apparemment à l'éviter (pas trop vite d'ailleurs) en leur donnant des cadeaux. Mais, pratiquement, cette parole est surtout désirée, et un hóron serait parfaitement déçu, au fond de lui-même, si à une cérémonie qu'il organise, les wóloso ne venaient pas jouer leur rôle parodique habituel. On peut donc se demander si le véritable moyen de pression de ces derniers ne tient pas finalement au besoin (et non à la peur) que les hóron ont de ce type de parole, et si les cadeaux qu'ils donnent ne sont pas plutôt une récompense pour un plaisir interdit.

70 Selon une telle hypothèse, les wóloso dònkili auraient en réalité une double fonction cathartique ; catharsis pour le producteur du message qui se libère ainsi partiellement de son agressivité de dominé, et catharsis pour le consommateur, qui peut vivre ainsi, par captifs interposés, les situations qui lui sont interdites.

71 Il ne faut certes pas exagérer la récupération des wóloso dònkili par la culture officielle dioula. Il arrive que ceux-ci soient produits dans des conditions non prévues par l'étiquette, notamment à l'occasion de réels conflits entre les captifs et les hóron, comme pour le dernier spécimen cité dans notre corpus. L'œuvre retrouve alors toute sa force subversive.

Par ailleurs, ces chants appartiennent à un genre véritablement marginal et relativement déconsidéré. Il y a chez les Dioula, un terme générique pour désigner l'ensemble des discours formalisés et institutionnalisés par la tradition, ce que nous appelons généralement la « littérature orale ». Il s'agit de l'expression kúma kóro qui signifie "parole ancienne». Nous avons recueilli à Kong, au cours de cinq années consécutives d'enquête un corpus de plusieurs milliers d'œuvres, qui a révélé quarantesept genres différents, selon les distinctions de la taxinomie autochtone. Nous avons mené une enquête auprès d'un échantillon de la population en lui demandant d'établir une hiérarchie de prestige entre tous ces types de parole. Les wóloso dònkili se retrouvent toujours dans les dernières places (y compris auprès des wóloso, ce qui montre qu'ils ont bien intégré les valeurs de la culture dominante), et un tiers environ des personnes interrogées a même refusé au genre le statut de kúma kóro. Ils restent donc à la frontière du champ littéraire, un peu comme ces productions que dans nos cultures écrites, nous nommons " paralittérature ».

Alors, les wóloso dònkili, " parole buissonnière »? Sans doute encore, oui, mais ce sont des herbes folles qui poussent tout de même dans les jardins bien taillés de l'institution. Et on peut faire confiance aux jardiniers hóron pour qu'elles n'envahissent pas trop les allées.

\section{NOTES}

1. Il peut arriver aussi, plus rarement, que la dénomination de genres de la littérature écrite fasse référence à des propriétés relatives aux conditions de l'énonciation, comme dans le cas où l'on parle, par exemple, de roman-feuilleton.

2. L'orthographe des mots dioula suit les conventions en usage pour la transcription des langues manding en Côte d'Ivoire. 
3. Síya, terme générique, signifie d'une façon générale, "sorte, genre, espèce ". Appliqué aux hommes, il peut désigner la race, sens qu'il prend dans le contexte de sa première apparition. Lorsqu'il intervient pour la deuxième fois, il spécifie plutôt la catégorie sociale représentée par les wóloso. Toute la force du chant en langue dioula provient justement du fait que c'est le même mot qui, grâce à sa polysémie, est utilisé dans les deux sens.

4. Les wóloso désignent les captifs de case, mais le terme générique pour définir la condition d'esclave est jòn dans la plupart des parlers manding.

5. béma désigne le grand-père, mais c'est aussi un terme d'adresse affectueux employé à l'égard de quelqu'un qui est plus âgé que le locuteur.

6. Nous avons volontairement traduit par des termes grossiers les mots wúlu (qui désigne le sexe masculin) et byغ̀ qui désigne le sexe féminin), afin d'essayer de respecter le niveau de langue original. Wúlu est en effet un terme grossier, considéré comme vulgaire pour désigner le pénis, comme fóro, sans doute encore plus cru (pine, bite), par opposition à ç̀ya (ou kàya), terme euphémique désignant la «masculinité » (sexe masculin, parties génitales). De même, byc̀ est un terme considéré comme impoli qui s'oppose à mùsoya (sexe féminin).

7. Curieusement on retrouve avec kàyakiri un terme poli. Nous avons donc changé de niveau de langue.

8. Selon l'avis même des interprètes de ce chant, il s'agit d'une onomatopée évoquant le bruit de la verge dans le vagin, à la fin des rapports, lorsqu'après l'éjaculation, elle commence à revenir à une certaine flaccidité.

9. La parodie des chants de wóloso ne tient pas seulement à la contrefaçon de l'énoncé, mais aussi au burlesque de l'énonciation. La façon de chanter en criant et de danser, de façon souvent obscène, peut être aussi un élément de la parodie.

10. Il s'agit ici bien entendu de l'oiseau (en dioula jùma- Loptoptilos Crumenifer), et non du maître coranique (kàramogo) couramment désigné par ce terme dans le français familier d'Afrique.

11. Le texte intégral de ce chant a déjà été publié en version bilingue par nos soins, « Le chant de Kurubi à Kong ", Annales de l'Université d'Abidjan, Traditions orales, série J, fasc. II (1978, 85-114).

12. Syé est réalisé [ $\left.\int j e ́\right]$. En fait [s] suivi de la fricative palatale [j] est toujours réalisé comme une chuintante [ [] . Dans l'orthographe, on le notera « $s$ ».

13. L'igname a des feuilles longues et minces, ce qui explique l'image.

14. C'est la fête dite du «mouton» que les musulmans, en souvenir du sacrifice d'Abraham, immolent le dixième jour du mois de pèlerinage (Sourate XXXVII, verset 107).

15. Expression idiomatique figurée signifiant mot à mot: "je coupe le village en deux », «j'ai parcouru la moitié du village ».

16. Le suffixe "bari ", très employé dans le dioula de Kong, est en quelque sorte un privatif; ainsi, màlobari (sans honte) signifie «malpoli »; dénworobari (sans accoucher d'enfant) s'emploie pour désigner une femme "stérile»; folibariya (le fait de ne pas saluer) exprime un type d'incorrection très grave chez les Dioula. Ici, « cet ignorant du bien » qualifie l'enfant qui vient de naître, et qui ne connaît rien des bonnes choses.

17. fórokiri, comme byc̀ et wúlu, appartiennent au registre grossier, les termes polis pour désigner les testicules étant bèlıkiri ou kàyakiri.

18. gbéndege: sorte de place appartenant à un quartier ou à un ensemble de cours où se déroulent les manifestations publiques concernant cette partie de l'agglomération.

19. Cf. par exemple, ce lásiri dònkili destiné à honorer la famille Baro, à travers le plus prestigieux de ses ancêtres, l'Alimami Baro: «Salut à toi, tueur de lion, Bara, salut à toi, tueur d'albinos, à qui on ne peut comparer le reste des Dioula. »

20. Cf. note 6 .

21. Tòngo désigne le bélier qui n'a pas de corne. On trouve parfois le terme utilisé dans l'expression plus complète et plus explicite sàgajigi tòngo « bélier sans corne ».

22. Cœur est à entendre ici au sens de courage. 
23. Pour bien comprendre cette pratique, il faut savoir que chez les Dioula, comme dans beaucoup de sociétés africaines, piler n'est pas seulement une opération utilitaire, mais aussi un acte esthétique dans lequel entrent à la fois la beauté du geste et la qualité du rythme produit.

24. Encore une onomatopée censée évoquer le bruit du coït.

25. Titre qu'on donne à ceux qui ont accompli le pèlerinage à La Mecque.

26. bilancE désigne à la fois le beau-père, le gendre, le beau-frère.

27. Ce qui se comprend du fait de la filiation patrilinéaire en cours chez les Dioula. Une famille hóron sera peu disposée à donner sa fille à un wóloso (sauf bien sûr s'il est devenu un notable de la Côte d'Ivoire moderne) qui lui donnera une descendance qui sera considérée elle-même wóloso.

\section{RÉSUMÉS}

Les chants de captifs dont il est question dans cet article parodient publiquement, en termes très grossiers, les chants cérémoniels et rituels de la culture officielle dioula concurremment à leur exécution. En première apparence, il s'agirait donc d'un genre subversif de la part des anciens captifs qui affichent ainsi leur insolence envers leurs anciens maîtres. Mais une analyse plus poussée de telles pratiques montre que les choses sont un peu plus compliquées et que, dans ces parodies rituelles, il y a aussi un échange de services implicite entre les ex-captifs et les hommes libres de naissance où chacun trouve son compte dans le cadre d'un certain équilibre social.

Prisoner songs publically parody, in very crude terms, the ceremonial and ritual songs of official Diula culture. At first sight, it seems we are dealing with a subversive genre performed by ancient captives who thus proclaim their insolence vis-a-vis their ancient masters. But in-depth analysis of these practices reveals that things are much more complicated and that in these ritual parodies, there is also an implicit exchange of services between the ex-captives and the men free by birth, where each finds what he needs within the framework of a certain social equilibrium.

\section{INDEX}

Index géographique : Afrique de l'Ouest

Mots-clés : chansons paillardes, captifs domestiques, subversion, catharsis

Thèmes : anthropologie (Afrique)

Keywords : Diula, Bawdy Songs, Domestic Captives, Subversion, Catharsis, Anthropology nomsmotscles Dioula 